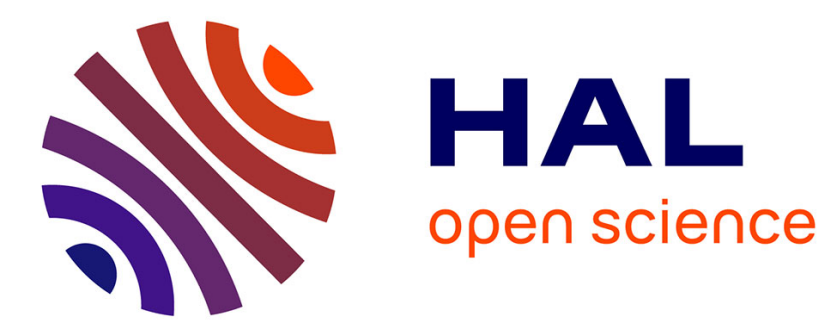

\title{
The Impact of Human-Robot Interfaces on the Learning of Visual Objects
}

Pierre Rouanet, Pierre-Yves Oudeyer, Fabien Danieau, David Filliat

\section{To cite this version:}

Pierre Rouanet, Pierre-Yves Oudeyer, Fabien Danieau, David Filliat. The Impact of Human-Robot Interfaces on the Learning of Visual Objects. IEEE Transactions on Robotics, 2013, 29 (2), pp.525-541. 10.1109/TRO.2012.2228134 . hal-00758241

HAL Id: hal-00758241

https://hal.inria.fr/hal-00758241

Submitted on 28 Nov 2012

HAL is a multi-disciplinary open access archive for the deposit and dissemination of scientific research documents, whether they are published or not. The documents may come from teaching and research institutions in France or abroad, or from public or private research centers.
L'archive ouverte pluridisciplinaire HAL, est destinée au dépôt et à la diffusion de documents scientifiques de niveau recherche, publiés ou non, émanant des établissements d'enseignement et de recherche français ou étrangers, des laboratoires publics ou privés. 


\title{
The Impact of Human-Robot Interfaces on the Learning of Visual Objects
}

\author{
Pierre Rouanet, Pierre-Yves Oudeyer, Fabien Danieau and David Filliat
}

\begin{abstract}
This paper studies the impact of interfaces allowing non-expert users to efficiently and intuitively teach a robot to recognize new visual objects. We present challenges that need to be addressed for real-world deployment of robots capable of learning new visual ;objects in interaction with everyday users. We argue that in addition to robust machine learning and computer vision methods, well-designed interfaces are crucial for learning efficiency. In particular, we argue that interfaces can be key in helping non-expert users to collect good learning examples and thus improve the performance of the overall learning system.

Then, we present four alternative human-robot interfaces: three are based on the use of a mediating artifact (smartphone, wiimote, wiimote and laser), and one is based on natural human gestures (with a Wizard-of-Oz recognition system). These interfaces mainly vary in the kind of feedback provided to the user, allowing him to understand more or less easily what the robot is perceiving, and thus guide his way of providing training examples differently.

We then evaluate the impact of these interfaces, in terms of learning efficiency, usability and user's experience, through a real world and large scale user study. In this experiment, we asked participants to teach a robot twelve different new visual objects in the context of a robotic game. This game happens in a home-like environment and was designed to motivate and engage users in an interaction where using the system was meaningful. We then discuss results that show significant differences among interfaces. In particular, we show that interfaces such as the smartphone interface allows non-expert users to intuitively provide much better training examples to the robot, almost as good as expert users who are trained for this task and aware of the different visual perception and machine learning issues. We also show that artifact-mediated teaching is significantly more efficient for robot learning, and equally good in terms of usability and user's experience, than teaching thanks to a gesture-based human-like interaction.
\end{abstract}

Index Terms-Human-robot interaction, user interfaces, robot learning, object visual recognition, user study, personal robotics.

\section{INTRODUCTION}

A. One challenge of personal robotics: learning from nonexpert humans

Personal robotics has been drawing an increasing amount of interest recently, both from an economic and a scientific point of view. Many indicators seem to show that the arrival of this kind of robot in our everyday homes will be one of the major events of the $21^{\text {st }}$ century [1]. In particular, they are predicted to play a key role in our aging society and especially in applications such as domestic services, telesurveillance or entertainment [2]. Yet, many challenges still

P. Rouanet, P-Y. Oudeyer, F. Danieau and D. Filliat are with INRIA and Ensta-ParisTech, France (see http://flowers.inria.fr/) need to be addressed before allowing personal robots to operate in our homes. They include a diverse set of questions related to perception, navigation, manipulation, learning, human-robot interaction, usability and acceptability. In this paper, we are more particularly interested in the transverse challenge: providing the robot with the ability to adapt itself to its environment through learning by interaction with nonexpert users. This is a key feature for the development of personal robotics. Indeed, unlike industrial robotics where the environment is very structured and known in advance, personal robots will have to operate in uncontrolled, unknown and/or changing environments. More importantly, they will have to interact with humans who may potentially have very diverse expectations and preferences. Thus, the robot should have the capacity to learn from non-expert humans.

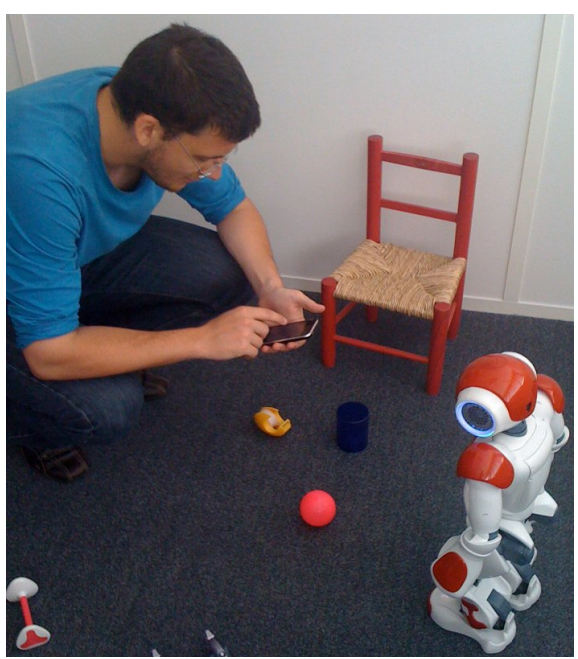

Fig. 1. Using a device as a mediator object between the human and the robot to control the movements of a personal robot allows non-expert users to teach it how to recognize new visually grounded objects.

\section{B. Studying the role of the interface for social robot teaching of new visual objects}

Techniques allowing robots to learn from interaction with humans have been widely explored in the literature, including approaches such as imitation learning and learning by demonstration (e.g. [3], [4]) or socially guided exploration (e.g. [5]). Having robots learn from humans requires both the development of machine learning algorithms (e.g. to encode and generalize new capacities) and the elaboration of intuitive and robust human-robot interaction techniques. While those 
two challenges are crucial, a large part of the work done in social learning focuses on the first problem [4][6][7]. Yet, the interaction mechanisms are known to play a key role in human teaching (e.g. [8]). Thomaz and Breazeal have shown the importance of understanding the human teacher/robotic student relationship in developing learning algorithms suited for social learning [5]. Calinon and Billard have proposed the development of learning by demonstration systems which take into account the interaction scenario [9]. Mechanisms such as joint attention have also been identified as crucial in social learning for both humans and robots teaching [10][11]. Furthermore, the importance of the role of interfaces and interaction becomes paramount when it comes to deploying robot learning systems outside the laboratory, where they shall be used by non-expert humans users.

In this paper, we focus on this latter issue and study the impact of human-robot interfaces allowing non-expert users to efficiently and intuitively teach a robot to recognize new visual objects. This is a case-study task bound to be needed in many future personal robotics applications. We present an integrated system which combines machine learning techniques, computer vision techniques, and various alternative humanrobot interfaces. The goal of the whole system is to allow a non-expert humans to show new visual objects to a robot (for which it does not already have a model and thus cannot segment easily) and associate a name so that it can be used as a training example allowing the robot to recognize these objects later (see figure 2). A strong constraint is that the system should be efficient and usable by non-expert users which will provide only very few training examples per object class. It is important to notice that by "visual objects" we are not only referring to actual physical objects (e.g. a ball) but to any region of an image having specific visual features. This very generic definition also includes more abstract objects such as a painting, stairwell or even an open door which should also be recognized by a personal robot.

As we will explain, a key challenge is that non-expert users typically have a wrong a priori understanding of what the robot sees or does not see, which can easily lead them to provide low quality training examples (e.g. examples where the objects they want to show to the robot is not even on the image perceived by its camera). We argue that the design of interfaces can be key in helping non-expert users to collect good learning examples and thus improve the performance of the overall learning system.

After detailing the related work in III we present in section III four alternative human-robot interfaces: three are based on the use of a mediating artifact (smartphone, wiimote, wiimote and laser) and one is based on natural human gestures (with a Wizard-of-Oz recognition system). These interfaces mainly vary in the kind of feedback provided to the users, permitting them to understand more or less easily what the robot is perceiving, and thus guide their way of providing training examples differently. As a consequence, as we will show, interfaces that provide the right kind of feedback can allow at the same time the human to understand what the robot is seeing at any given moment, and vice versa the robot can infer efficiently what the human is trying to show to him. This form of synchronization of what each other is looking at, and made possible by particular kinds of user interfaces, is an elementary form of joint attention ${ }^{1}$ which has been shown to be crucial in the the field of developmental robotics for teaching new visual concepts to a robot [11][12]. Our work is thus located at the crossover of three important research domains: social learning in robotics [4], human-robot interaction [13] and developmental robotics [14].

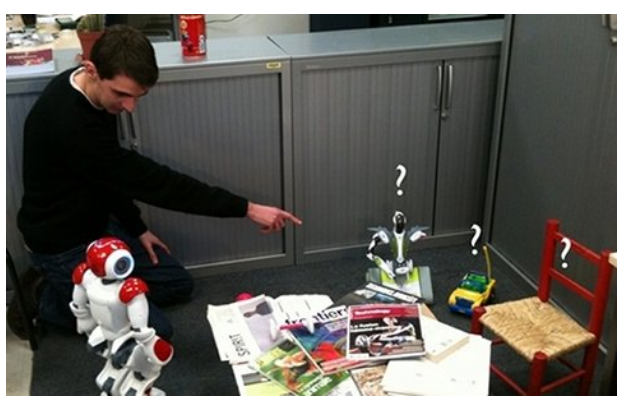

Fig. 2. To allow users to designate a particular object to a robot in a cluttered environment, we need to provide them with a robust and accurate pointing detection. Otherwise it may lead to restrictive interaction and even to false learning examples.

We then evaluate and compare in section IV the impact of these interfaces, in terms of learning efficiency (robot's point of view) and usability and user's experience (user's point of view), through a real world and large scale user study (107 participants). In this study, which took place in a science museum in Bordeaux, we asked participants to teach a humanoid robot $\mathrm{Naq}^{2}$ twelve different new visual objects in the context of a robotic game. This robotic game happened in a home-like environment and was designed to motivate and engage users in an interaction where using the integrated system was meaningful.

We chose to follow a very standard user-centered approach to design our interfaces based on mediator objects as we wanted our system to be effectively usable by non-expert humans in plausible interactions, i.e. outside of the lab, with a personal robot. With such an approach we first analyzed the context of use, then conceived the interface and finally we evaluated it. This cycle was repeated until the specified requirements were matched. In this paper, we are presenting the last complete iteration of our development. While some earlier versions of subparts of our integrated system have already been presented in [16] [17] [18] [19], they have been

${ }^{1}$ It is an elementary form of joint attention in the sense that both agents can infer what the other is looking at and/or perceiving, without an explicit cognitive model of attention, see [11]

${ }^{2}$ The Nao robot represents, in our opinion, the current personal affordable robots well, with a humanoid appearance. Furthermore, we choose to use it as an autonomous robot, i.e. with only onboard sensors, and not to enhance its capacities with external devices such as cameras fixed on the ceiling such as those used in smart environments or ubiquitous robotics approaches [15]. We argue that the complexity of this kind of installation could prevent their use in everyday homes in the near future. Second, it is important to note that this kind of system, while improving the perceptual capacities of the robot will not fundamentally change the attention problem that we are trying to tackle here. For instance, a pointing gesture will remain ambiguous in a cluttered environment even with the use of fixed cameras. 
modified and improved since then and they are here presented as an integrated system for the first time.

We then discuss results that show significant differences among interfaces. In particular, we show that interfaces such as the smartphone interface allows non-expert users to intuitively provide much better training examples to the robot, almost as good as expert users who are trained for this task and aware of the different visual perception and machine learning issues. We also show that artifact-mediated teaching is significantly more efficient for robot learning while better in terms of usability than teaching using gesture-based human-like interaction.

Finally, a discussion of the main results and of our design choices is presented in the section $\mathrm{VI}$

\section{RELATED WORK}

The classification and recognition of new visual objects have been studied intensely from visual perception and machine learning perspectives. Many approaches, such as the bags of visual words we are using in this paper, have been recently developed [20][21][22]. Those learning systems are highly efficient when trained with a large database of good labeled examples (see PASCAL VOC for instance [23]). Yet, to solve this problem in a real human-robot interaction scenario: i.e. outside of the laboratory, with non-expert users in a realistic use scenario, one needs to tackle a crucial issue not addressed in the machine learning and computer vision literature: how to collect good training examples through relatively few but intuitive interactions with non-expert users? And, how to collect examples by using current social robots which typically have limited sensors and, in particular, a strongly constrained visual apparatus? Those questions are addressed in this article.

The questions of drawing a robot's attention, pointing toward objects and realizing various forms of joint attention to teach the name of new objects have also been widely studied. For instance, they are closely related to research done in robot language acquisition and in particular the construction of visually grounded lexicons [24][25][26]. Yet, in this literature most authors are focusing on the perception and machine learning questions. In particular, as they try to model human language acquisition, they choose to directly transpose the human-like interactions to human-robot interactions to allow humans to show new associations between visual objects and their names. For instance, Kaplan developed a complete social framework based on human-like interactions such as pointing gestures and speech recognition to allow users to teach words associated with objects to an AIBO robot [27]. Scasselati used pointing gestures and gaze tracking to draw a robot's attention [28]. In this work, he used a fixed upper-torso and thus constrained the interaction. Pointing gestures have also been used to guide a robot companion [29][30].

Unfortunately, existing associated techniques for gesture, gaze and speech recognition and interpretation are not robust enough in uncontrolled environments (due to noise, lighting or occlusion) and most social robots have a body whose shape and perceptual apparatus is not compatible with these modes of interaction (low quality and noisy sensor, small angle of view, small height...). Thus, these a priori intuitive systems have to be used by expert users in the sense that they have to understand the limitations of the robot in order to behave according to a very restrictive protocol which will allow the interaction to work. One way to circumvent this problem is to have a very controlled setup. For instance, Roy presented a framework that allows a robotic system to acquire visually grounded words [31]. Here, users have to place objects in front of the robot and then describe them. We argue that this kind of experiment cannot be directly transposed into a real world application in personal and social robotics with non-expert users.

Yet, as personal robotics is predicted to become commonplace in our home environments in the $21^{\text {st }}$ century, it is really important that even non-expert users can robustly designate objects to their social robot in an uncontrolled environment. We should provide interfaces which are intuitive in order to avoid misunderstanding or frustration during interaction but also to help users collect good learning examples. Indeed, in a cluttered environment, non-robust pointing may lead to the designation of the wrong object and thus completely incorrect learning examples which will decrease the performance of the whole learning system. In their work, Kaplan and Steels identified the lack of robustness in the interface as a major limitation of their system and they showed that the lack of robustness of the interface often leads to a number of bad learning examples [32].

Another widely used way to tackle this pointing and joint attention problem is to allow users to directly wave objects in front of the camera of the robot [33][34]. Thus, we can ask the robot to always focus its attention on the moving objects. Furthermore, it also allows the separation of the object from the background by subtraction of the motionless part of the scene. However, with this technique, users can only show to the robot small and light objects which can be easily carried as they will have to be waved in front of the robot. Thus, we can not show objects such as a table, a plug or a painting on a wall. Moreover, for the elderly or the disabled waving objects could be really tiring or even impossible.

We argue that one way to help achieve some of the abilities described above intuitively and robustly without facing the problems encountered when waving objects, is to develop simple artifacts that will serve as mediators between the human and the robot to enable intuitive communication. Interfaces based on mediator objects have already widely been used in the domain of human-robot interaction and especially to draw a robot's attention toward an object. For instance, Kemp et al. used a laser pointer to easily and robustly designate objects to a robot in order to ask it to fetch them [35]. Here, they used the laser pointer as a point-and-click interface. They showed that inexperienced participants managed to correctly designate objects to a robot. Furthermore, thanks to the laser spot light, the human can also accurately know what he is pointing at. Yanco et al. used an interface based on an input device (touch screen or joystick) to select objects which will be grasped by a wheelchair mounted robotic arm [36]. In their work the user can directly monitor the object selection on the screen of the device. As in our system, they can both draw the robot's attention toward objects and so realize joint attention 
between the human and the robot. However, their robot is able to automatically grasp the object from a detected 3D spot in a framework that requires an image segmentation algorithm and/or a priori object knowledge. If objects are not known beforehand these are still difficult problems.

Other mediator object based interfaces have been developed recently. For instance, Fong et al. used a PDA for remote driving [37], and Kaymaz et al. used it to tele-operate a mobile robot [38]. Sakamoto et al. showed how they can control a house cleaning robot through sketches on a Tablet PC [39]. Ishii et al. proposed a laser pointer-based interface where users can draw stroke gestures using the laser to specify various commands such as path definition or object selection with lasso gestures [15]. However, in their work they used calibrated ceiling-mounted cameras and vision-based ID tags to circumvent object recognition issues. Yet, to our knowledge, nobody has used this kind of interface for interactions that involve robot teaching, such as teaching new words for new visual objects.

\section{OUTLINE OF THE SYSTEM}

As explained above, we present here an integrated system to allow non-expert users to teach a personal robot how to recognize new visually grounded objects in real world conditions. In particular, this means that our system should allow a user to draw the robot's attention toward an object present in its surrounding and then collect a learning example of it. The robot could thus recognize and search for an already taught object later on. In this version of the system, labels are automatically associated with images. We will describe in the section VI, a more advanced version of our system which allows users to associate new acoustic words to the visual objects.

This system has to deal with visual perception, machine learning and interaction challenges. The visual perception and machine learning parts of our system are based on a version of the advanced bags of visual words technique [21]. These computer vision and machine learning algorithms have been chosen because, to us, they represent robust and standard tools often used as a baseline to compare with more recent techniques. Furthermore, we are here focusing on the four different interfaces notably developed to tackle the pointing and attention challenges. Three interfaces are based on mediator objects while the last one is based on arm and hand gestures with Wizard-of-Oz recognition.

Our system was embedded in the Nao robot designed by the company Aldebaran Robotic ${ }^{3}$. The robot was only used here to collect the learning examples (i.e. take the pictures) and store them. The actual learning was performed offline on a computer. We have already explained why we chose this particular robot and used it as an autonomous robot. The implication of this choice will be discussed later.

\section{A. Visual perception}

We adopted the popular bags of visual words approach [20] to process images in our system. This method was developed

\footnotetext{
3 http://www.aldebaran-robotics.com/
}

for image categorization and object recognition and relies on a representation of images as a set of unordered elementary visual features (the words) taken from a dictionary (or code book). The term "bag of words" refers to text document classification techniques that inspired this approach where documents are considered to be an unordered sets of words. In its basic implementation that we use here, a classifier predicting the object identity is based on the occurrence frequencies of the visual words in an image, thus ignoring any global image structure. There are several extensions which introduce some global geometry in order to improve performance (e.g. [40]), but these extensions were not necessary to implement in order to demonstrate the interest of the interfaces which is the subject of this paper. Several applications also exist for robotics, notably for navigation (e.g. [41], [42]).

The words used in image processing are based on automatically detected local image features. The feature detectors used are usually invariant to image rotation, scale and partially to affine deformation so as to be able to recognize objects under varying point of view. Among the many existing feature detectors, we chose SURF [43] for its performance and reasonable processing cost. For each detected feature, a descriptor is computed that encode the local image appearance. A dictionary is created by clustering a large set of feature descriptor extracted from images representative of the environment. In our implementation, we use a hierarchical k-means algorithm to create a tree-structured dictionary that enable fast word look up [44]. The size of the dictionary was set to $2^{12}$ in our experiments.

This model has interesting characteristics for our application: the use of feature sets make it robust to partial object occlusions and the feature space quantization brings robustness to image noise which is linked to object position, camera noise or varying illumination.

\section{B. Machine learning}

For our application, the classifier designed for object recognition should be trained incrementally, i.e. it should be able to process new examples and learn new objects without the need to reprocess all the previous data. To achieve that, we use a generative method in which training entails updating a statistical model of objects, and classifying involves evaluating the likelihood of each object given a new image.

More specifically, we use a voting method based on visual words occurrences for each object. The recorded statistics during learning (according to the learning method described later) are the number of occurrences $O_{w o}$ of each visual word $w$ of the dictionary in the training examples of each object $o$. For object detection in a new image, we extract all the visual words from this image and make each word $w$ vote for all objects $o$ for which $O_{w o} \neq 0$. The vote is performed using the term frequency-inverted document frequency (tf-idf) weighting [20] in order to penalize the more common visual words. The recognized object is the one with the best vote.

Estimating the statistics $O_{w o}$ requires the labeling of examples with their associated object name. The quality of object recognition is obviously strongly influenced by the number and 
quality of training images [45]. In computer vision, creating good image datasets is therefore an important aspect, to which a large amount of time is devoted, but this time is not available when interactive learning takes place with a robot for new objects as performed in our work. Moreover, precisely selecting relevant training images is also not always possible, depending on the interface used to control the robot. As will be described later, we will use two methods for labeling based on the information given by the user: labeling the whole image or labeling only an image area (given by the user) that represents the entire object. Then we will show the influence of these methods on final object recognition.

\section{Human-robot interaction}

In this section, we present the different interfaces developed. They were chosen to span the variety of mediator interfaces that one can imagine but also to explore the different kinds of feedback of what the robot is perceiving that can be provided to the users. Three of the interfaces are based on mediator objects such as the iPhone, the Wiimote or the laser pointer. We chose rather well-known and simple devices so users can quickly learn how to use them. The fourth interface was added in order to compare the mediator based interfaces to a humanlike interaction which, as we will demonstrate reveals itself to be less usable and less efficient than the mediator based interfaces.

In order to be compared fairly, each of these four interfaces has to provide the users with the exact same following abilities:

- driving the robot

- drawing its attention toward a direction or a specific object

- defining the object area inside the image (only the iPhone and Wiimote laser interfaces provide this ability; for the two other interfaces the whole image was taken into account in the evaluation)

The mediator objects were not used to trigger the capture of a new learning example. Instead, when users think that the robot sees the object they want to teach they had to directly touch its head. We chose to force this physical interaction with the robot in order to increase the feeling of collaboration. Yet, the different mediator objects could easily be adapted to directly trigger the capture.

It is important to notice that all the interfaces were based on the exact same sensorimotor capacities and functionalities of the Nao robot. As argued before, the Nao sensorimotor apparatus represents well the present form of existing social robots to us. We also voluntarily choose not to enhance its capacities by using a ceiling or a wide range camera although it may have improved the usability of our interfaces. Indeed, as discussed in detail in section VI such improvement would not have solved the fundamental attention problems that we are trying to tackle here.

In the next sections, we will describe each interface in detail and emphasize their specificities. We chose to focus on three interfaces, which seemed the most interesting to us, but other mediator objects or interaction metaphors could have been imagined in this context (e.g. using a pico projector to display the field of view of the robot could have helped knowing what the robot could see).

\section{D. iPhone interface}

The first interface is based on an Apple iPhone used as a mediator object between the human and the robot We chose this device because it allows the display of information on the screen to the user and also allows interaction through intuitive and standard gestures. In addition, the multi-touch capacity provides numerous possibilities. Due to the large success of the iPhone we can take advantage of a familiar interface, allowing ease of use.

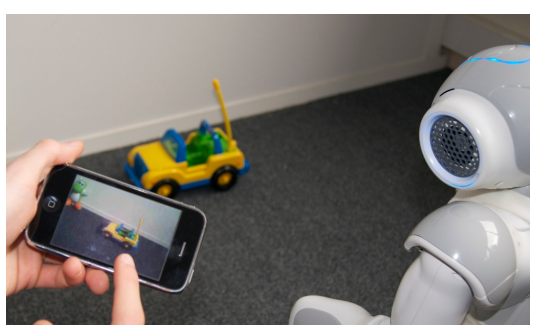

Fig. 4. We display the video stream of the camera of the robot on the screen. This allows accurate monitoring of what the robot is seeing.

In this system, the screen of the handheld device displays a continuous video stream of the robot's camera. It accurately shows what the robot is looking at, which can be monitored by the user which resolves the ambiguity of what the robot is really seeing (see figure 47. However, the user's attention is split into direct and indirect monitoring of the robot which may lead to the increase of the user's cognitive workload. Finally, having visual feedback seems to entertain the user while the robot is moving as shown in pilot studies [16].

When the human wants to draw the robot's attention toward an object, which is not in its field of view, the user can sketch on the screen to make it move to an appropriate position: vertical strokes for forward/backward movements and horizontal strokes for right/left turns. Elementary heuristics are used to recognize these straight touch gestures. The moves of the robot are continuous until the user re-touches the screen in order to stop it. Pointing on a particular point on the screen makes the robot look at the corresponding spot (see figure 5). This is a very convenient way of drawing the robot's attention toward a specific object.

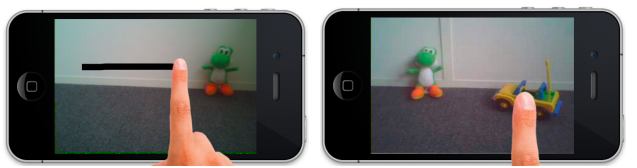

Fig. 5. Drawing attention toward an object: the user first sketches directions to position the robot such that the object is in its field of view (left), and if he wants to center the robot's sight on a specific spot the user can just tap on the screen (right).

As explained above, when the user wants to show an object in order to teach a name for it, he can first make sure that

\footnotetext{
4 http://youtu.be/vrMsalj2SDM
} 


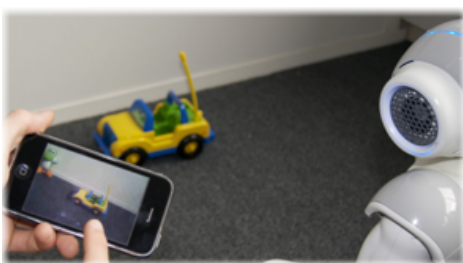

(a) draw the attention toward an object

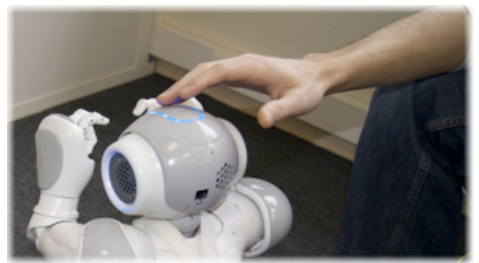

(b) trigger the capture

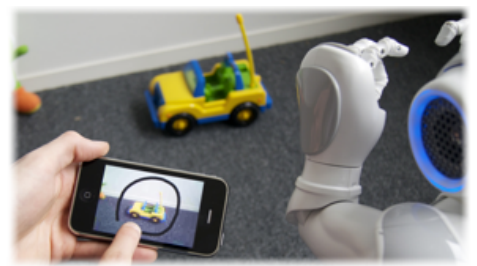

(c) encircle the area of the object

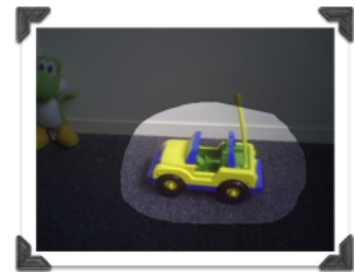

(d) the new learning example

Fig. 3. To make the robot collect a new learning example, users have to first draw the robot's attention toward the object they want to teach through simple gestures. Once the robot sees the object, they touch the head of the robot to trigger the capture. Then, they directly encircle the area of the image that represents the object on the screen. The selected area is then used as the new learning example.

the object is in the field of view of the robot by monitoring whether the object is displayed on the screen or not. Once he is certain that the robot sees the object, he touches the head of the robot to ask it to take a picture. Then, the system asks the user to sketch a circle around this object directly on the touch screen (as shown on figure 6. Circling is a really intuitive gesture because users directly "select" what they want to draw attention to. This gesture is particularly well-suited to touch-screen based interactions. For instance, Schmalstieg et al. used the circling metaphor to select objects in a virtual world [46]. Hachet et al. used 2D circle inputs for easy 3D camera positioning [47]. As for the straight strokes, heuristics are used here to recognize circular touch gestures based on the shape of the stroke and the distance between the first and the last point of the gesture. Circling is crucial to the robot since it provides a rough visual segmentation of the object which is otherwise a very hard task in unconstrained environments. With the stroke and the background image, we can extract the selected area and define it as our object's image. Classical computer graphics algorithms are used to compute this area (Bresenham line drawing and flood fill). The complete sequence of actions needed to provide a new learning example is summarized in the figure 3 .

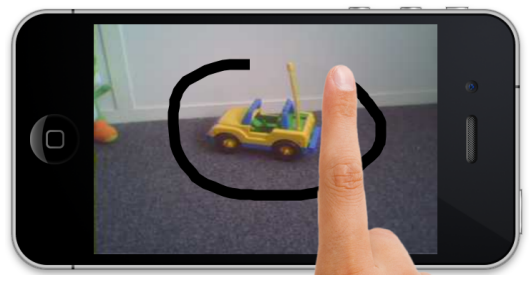

Fig. 6. Once the user asks the robot to take a picture of a new object, he can directly encircle it and thus providing a useful rough object segmentation.

\section{E. Wiimote interface}

The second interface is based on a Wiimote device (see figure 7. 5 The users can press one of the buttons of the arrows to move the robot. We use the very common flying vehicle metaphor: if we want to make the robot move forward, we keep the up arrow pressed and as soon as the button is released the robot will stop. This technique permits easy driving of the robot or the ability to draw it's attention toward a general direction. To aim the head of the robot, users have to orient the Wiimote: i.e. we directly map the values of the accelerometers to the pan/tilt values of the robot's head. Thus, users can always focus their attention on the robot.

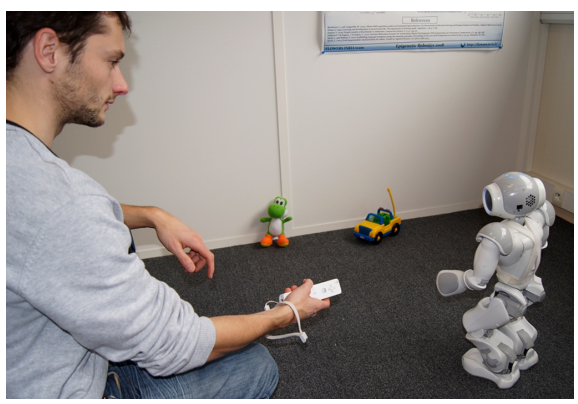

Fig. 7. Users can move the robot by using the directional cross or directly orienting the Wiimote to aim its head. However, the lack of feedback makes it very difficult to estimate whether the robot really sees the object the user wants to teach.

However, this interface does not provide any feedback about what the robot is perceiving. In particular, users can not be sure whether the robot sees an object or not. So, they have to "guess" what the robot really sees, which can be a very difficult task as illustrated in the experiments presented below.

\section{F. Wiimote and laser interface}

In this third interface, the Wiimote is also used to drive the robot. However, as shown on figure 8, a laser pointer is combined with the Wiimote and used to draw the robot's attention 6 .

The robot is automatically tracking the laser spot and aims its head in order to keep the spot near the center of its sight. We chose a laser pointer as the interaction device as this method to draw someone's attention is quite common in our every-day life, in oral presentations for instance, and so is an intuitive interaction for users. Here, users can draw the robot's attention toward a direction by smoothly aiming its head toward the right direction or they can point to a particular object directly once inside the robot field of view, by designating it with the laser pointer.

We automatically detect the laser spot in the images received from the camera of the robot. We used a very bright laser 


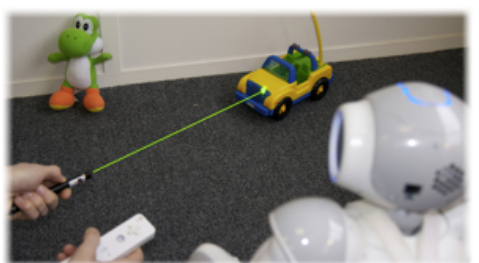

(a) draw the attention toward an object

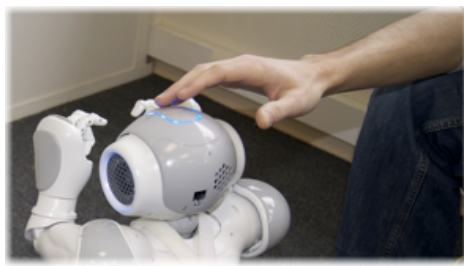

(b) trigger the capture

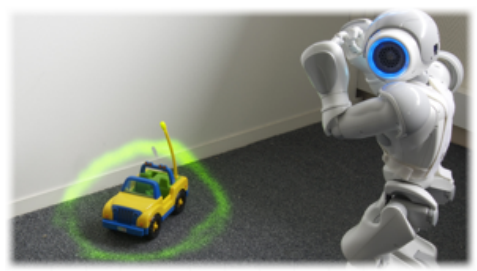

(c) encircle the area of the object

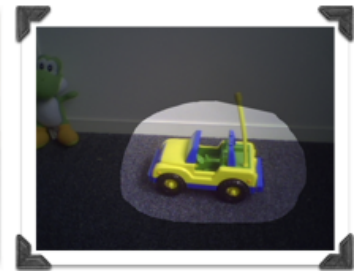

(d) the new learning example

Fig. 9. With this interface users can draw the robot's attention with a laser pointer toward an object. The laser spot is automatically tracked by the robot. They can ensure that the robot detects the spot thanks to haptic feedback on the Wiimote. Then, they can touch the head of the robot to trigger the capture of a new learning example. Finally, they encircle the object with the laser pointer to delimit its area which will be defined as the new learning example.

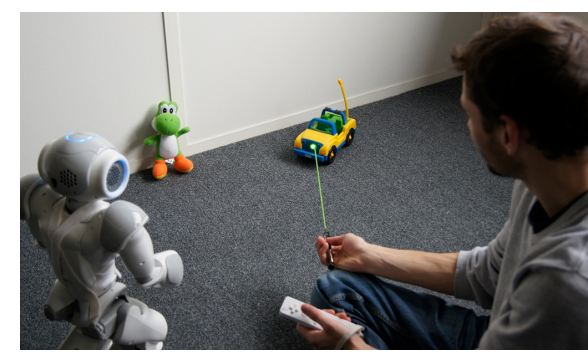

Fig. 8. Users can drive the robot with a Wiimote and draw its attention toward an object by pointing it with a laser pointer as the robot is automatically tracking the laser spot.

pointer with a significant spot size. We chose a green pointer, because "laser" green is probably a color less present in the everyday environment and so much more salient.

Unlike Kemp et al. who used an omnidirectional camera [35], or Ishii et al. who used ceiling-mounted cameras [15], in our work the field of view of the robot is very limited. So, drawing the robot's attention requires that the user correctly estimates the field of view of the robot. This can be a difficult task as non-expert humans are often prone to assume that the robot has a field of view which corresponds to a human one, while it is not the case most of the time.

We provide different feedback to help users better understand when the robot is detecting the laser spot and thus correctly estimate the field of view of the robot. First, as the robot is tracking the laser, users can monitor the movements of its head so that they have visual feedback. Second, we also provided haptic feedback by vibrating the Wiimote each time the laser spot was detected by the robot. With the combination of these two feedbacks, users know whether the robot was detecting the laser spot or not and can make sure that the laser pointer, and thus nearby objects, are in the field of view of the robot.

The feedback for this interface is more restricted than the complete feedback that is provided by the iPhone interface where users can directly monitor what the robot sees. Furthermore, users can not be sure that the robot sees the object they are designating entirely. They can only be sure that the robot is detecting the visible spot on a part of the object. For instance, when it is not possible to center the robot's head on the laser spot due to the robot's physical limit, a part of the object may be outside of its field of view.

The laser also provides visual feedback to the user. Indeed, the laser spot allows users to monitor what they are really pointing at and thus they can adjust their pointing if needed. This is particularly important in a cluttered environment where small deviations may lead to pointing to the wrong object leading to an incorrect learning example.

Once users manage to draw the robot's attention toward an object and trigger the capture of a learning example by touching the head of the robot, they can then encircle the object directly with the laser pointer (see figure 9 for the whole sequence). To record the encircling gestures done by the user, we store the detected points during these movements. Yet, as the frame rate of the camera is low and as the speed of the movement may really vary from one person to another, encircling once was not always enough to compute a reliable stroke. So, we asked participants to encircle the objects many times. All the detected points are recorded without keeping any structure information and stored in a point cloud. It is then fitted on an ellipsis, as the shape of encircling gestures tends to be elliptic. Finally, the robot indicates through a head movement whether it has detected enough points to compute a reliable ellipsis. The ellipsis is computed as follows [48]:

Based on the implicit equation of an ellipsis we can obtain the following system:

$$
\begin{gathered}
A x^{2}+B x y+C y^{2}+D x+E y+F=0 \text { with } A \neq 1 \\
\Rightarrow B x y+C y^{2}+D x+E y+F=-x^{2}
\end{gathered}
$$

Written in a matrix form:

$$
\alpha=\left(\begin{array}{ccccc}
x_{1} * y_{1} & y_{1}^{2} & x_{1} & y_{1} & 1 \\
\ldots & \ldots & \ldots & \ldots & \ldots \\
x_{n} * y_{n} & y_{n}^{2} & x_{n} & y_{n} & 1
\end{array}\right) \beta=\left(\begin{array}{c}
-x_{1}^{2} \\
\ldots \\
-x_{n}^{2}
\end{array}\right)
$$

As this system is overdetermined, we try to find the $X$ which best fits the equation in the sense of the quadratic minimization problem (least squares):

$$
\underset{X}{\arg \min }=\|\beta-\alpha X\|^{2}
$$

which is equivalent to solve the equation:

$$
\hat{X}=\left(\alpha^{T} \alpha\right)^{-1} \alpha^{T} \beta
$$

Once the ellipsis has been computed, we can use it to delimit the boundary of the object and thus roughly segment the image. In opposition to the iPhone interface where the 
encircling is done on the 2D image, here users encircle directly on the 3D environment. This could lead to projection issues, especially when the background is not planar. As we can see in the examples in figure 10, the projected stroke could sometimes "cut" the object and thus decrease the quality of learning examples.
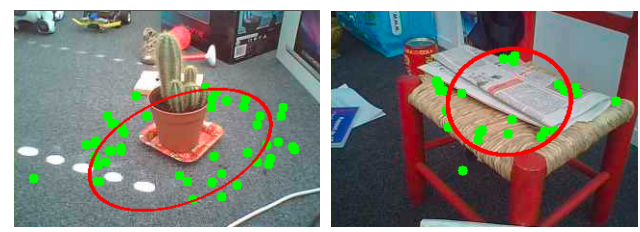

Fig. 10. Encircling with a laser pointer raises difficulties mostly due to the projection of the laser spot in the plane of the camera.

\section{G. Gestures based interface with WoZ}

In this last interface, users can guide the robot by making hand or arm gestures $7^{7}$. As we wanted to keep this interface as intuitive as possible we did not restrict the kinds of gestures that users can make. However, as gesture recognition is still a complex task, we used a Wizard-of-Oz framework where a human was controlling the robot according to different gestures the magician recognized. We can thus ensure that the recognition of the different gestures was not a limitation of this interface. As stated above we did not want to enhance the robot's capacities with a ceiling or wide-angle camera as we wanted to study a human-like interaction between non-expert users and what we think represent a typical actual personal robot well. Thus, all the four interfaces are based on the same robot sensorimotor capacities which allows a comparison of the different interfaces on a fairer basis. Furthermore, as explained in the discussion, even if using external sensors would probably improves the usability of this interface it would not fundamentally change pointing or attention issues.

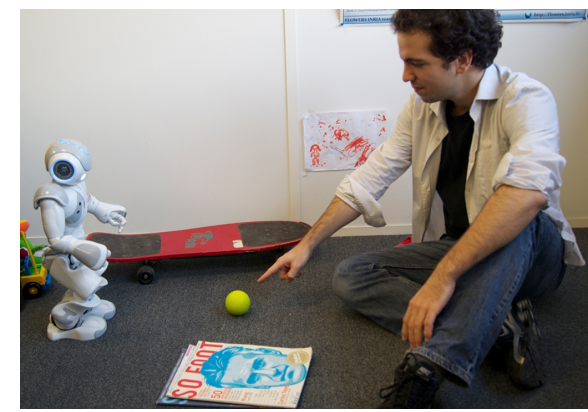

Fig. 11. In this mode of interaction, the robot is guided by the hand and arm gestures made by the user. In order to have a robust recognition, we used a WOZ framework, where the wizard was only seeing the interaction through the robot's viewpoint.

As the Wizard was only seeing interaction through the robot's eyes and therefore through a very limited visual apparatus, most of the gestures made by the users were outside of the robot's field of view. As a consequence, and as we

7 http://youtu.be/15GOCqXdgQg will show in the experiments below, even such a well-known interface with human-level intelligence may in fact lead to fragile interactions due to the differences between the robot's sensorimotor apparatus and the human's ones.

Obviously, this interface embeds a strong difference with the others. Indeed, the Wizard is a human who already has knowledge and strong biases about what may constitute an object shown to him, i.e. the object segmentation problem is here automatically solved by the human. Thus, when the object was pointed at, the wizard naturally centered the sight of the robot on it. Yet, on the other hand, in spite of this advantage when compared to other interfaces that are coupled with an autonomous robot, this interface does not perform so well as we will demonstrate.

Although this interface embeds some strong differences with the other, it still appears very interesting to us, as it first allows the investigation of a human-like interaction with an autonomous personal robot with a limited visual apparatus if we assume a human-level recognition and interpretation of gestures. Second, it also permits the comparison of our interfaces based on mediator objects with a human-like interaction and showed that because of the particular visual apparatus of the robot, this interaction may lead to a more restrictive and thus less satisfying interaction for users.

\section{EXPERIMENTAL EVALUATION WITH NON-EXPERT USERS}

As previously explained, we want here to study the impact of interfaces on robot learning of new visual objects through non-expert user teaching and in a real world home-like environment. This impact is evaluated along two main dimensions:

- Learning Efficiency: We test the quality of training examples collected by the robot through human guidance. This quality is evaluated both qualitatively (see below) and quantitatively through a measure of accuracy in classification in generalization (i.e. on images not in the collected dataset). We also want to study more specifically how encircling can impact the performance of the learning system.

- Usability and user's experience: We study below how intuitive, effortless and entertaining our interfaces are for non-expert users.

We argue that potential future users of social robots will not necessary be expert users, in the sense that they should be able to interact with their social robot without any prior specific training. Thus, it is crucial that our study is not a laboratory study but a real world study. In particular, we want to create a plausible context of interaction and have representative nonexpert participants, in order to preserve the ecological validity of our results and avoid the classical pitfalls of the evaluation in the HRI domain [49][50].

However, as those users have probably never interacted with a social robot before, asking them to show and teach objects to a robot is still an unusual and artificial task as shown by pilot studies [16][17][51]. Therefore, we need to embed this task in a scenario in order to justify it. In particular, we need to encourage the users to collect high quality learning examples. 
Moreover, we want a scenario that can entertain and maintain the user's motivation during the whole experiment. Finally, we want to conduct a large scale study and so we need to design a formal and reproducible experiment.

\section{A. A Robotic Game Experiment}

We argue that one solution to tackle the above mentioned issues was to design our user study as a robotic game. Games are well known to be a powerful way of captivating and engaging users through their storyline. For instance, serious games have been widely used for education or training, allowing learners to experience situations that are impossible or hard to reproduce in the real world [52]. We think that, in the same way that video games have managed to make novice users solve complex and unusual tasks by using mechanisms such as tutorials or briefings, we could design a robotic game experiment that helps users to better understand and remember all the steps needed to teaching visual objects to a robot. The scenario of the game also permits us to justify this artificial task. Finally, presenting the experiment as a game allows us to attract a wide and varying panel of participants. Participants would feel more comfortable participating in a game than a scientific experiment.

Thus, we created a game scenario to try to match all of the above. The users were told the following story: a robot, which has come from another planet, has been sent to Earth in order to better understand what seems to be a popular human habit: "playing football". Indeed, from their remote home, the robots have just picked up partial information about this practice and so they want to investigate further. Therefore, one robot was sent to the living room of a football fan to gather more clues. As the robot was damaged during its journey, it could no longer fulfill its mission alone. So, you will need to help it! The user was asked to help the robot to collect clues (i.e. collect learning examples of four different objects related to football). Every time the robot collected a new example, a false and funny interpretation of what the object can be used for was given by the robot.

\section{B. Experimental setup}

1) Game Environment: We recreated a typical $10 \mathrm{~m}^{2}$ living room located next to the café of a science museum in Bordeaux, France. We arranged furniture such as tables or chairs and many other various everyday objects (newspaper, plush toys, posters, etc...) in order to make it look inhabited. Among these various objects, 12 were directly related to football (see figure 12,. Those objects were the possible clues the robot needed to collect. They were chosen because they fit well within the scenario but also because they were textured and big enough so they could be robustly recognized by classical visual recognition algorithms (if provided with good quality learning examples). Other usual objects were added to the scene to make the environment cluttered (see figure 13 .

The design of the game environment had three main purposes:

- Reproduce a daily life area to provide participants with a stressless environment and to reduce the feeling of being evaluated.

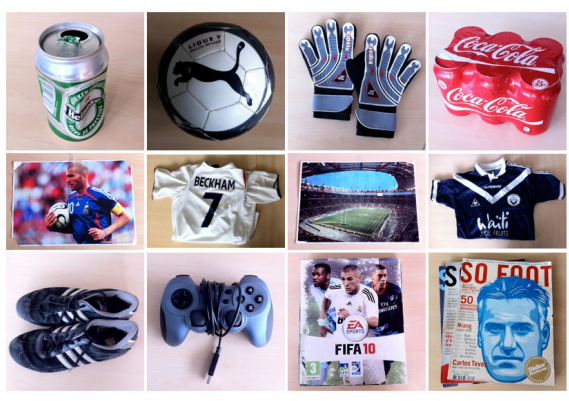

Fig. 12. For the experiment, we used 12 textured objects directly related to football: beer, ball, gloves, coke, a poster of Zidane, a jersey of Beckham, a poster of a stadium, a jersey of the Bordeaux team, shoes, a gamepad, a video game and magazines. Each participants had to teach four randomly chosen objects to the robot to help it better understand football.

- Conduct the experiment in a realistic environment, so users have to navigate the robot through a cluttered area and to collect real world learning examples (lighting conditions, complex background, ...).

- Immerse users in the scenario.

The global structure of the room remains unchanged during the whole experiment in order to get a constant test environment. Nevertheless, the small objects were randomly arranged every five experiments. Indeed, in a real home, while big objects such as furniture will not move, most of the small objects will often be moved and thus must be recognized in spite of their background.

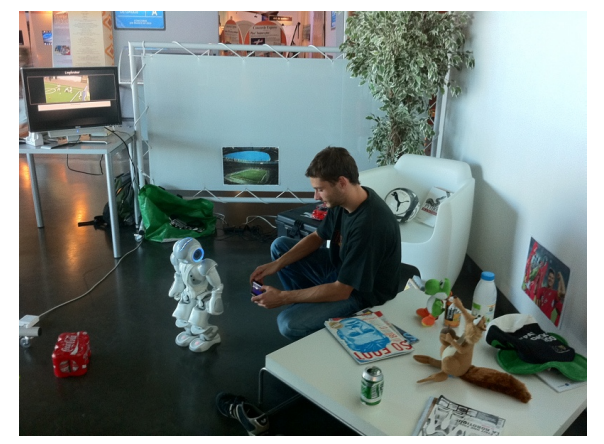

Fig. 13. The real world environment designed to reproduce a typical living room. Many objects were added in the scene in order to make the environment cluttered.

2) Robot: As stated previously, we used the Nao for our experiment. To make it more lively, we developed some basic behaviors such as yawning or scratching its head if the robot was idled for a long time. We also used different colors for its eyes to express simple emotions or to provide feedback to the users (see figure 14). Moreover, we added organic sounds to express the robot's mood.

3) Game Interface: As explained above, the design of our robotic game was inspired from a classic video game. We used a large screen as the game interface to display information to users such as cutscene video explaining the story.

This interface was also used to recreate a tutorial where participants learn one ability at a time: walking straight, turning, aiming the head of the robot and collecting a learning 


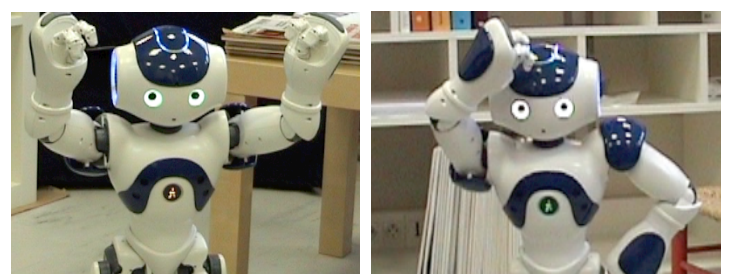

Fig. 14. Behaviors, such as "happy" (on the left) or scratching its head (on the right), were designed to make the robot look more lively and help the users better understand its behavior.

example. For each step, a short video explained how to realize the task with the interface they were using. After the video, the user was asked to effectively complete the task. Once they succeed, they could move on to the next stage. These videos were also a way to make users know the robot better. The final objective of the tutorial was to first collect a learning example which, in fact, was a picture of the user's face. The whole tutorial lasted about five minutes on average. After the tutorial, the real mission was explained to the participants via another video similar to the one in figure 15 . Thus, the game interface allowed us to present the whole experiment (both tutorial and mission parts) in one single game.
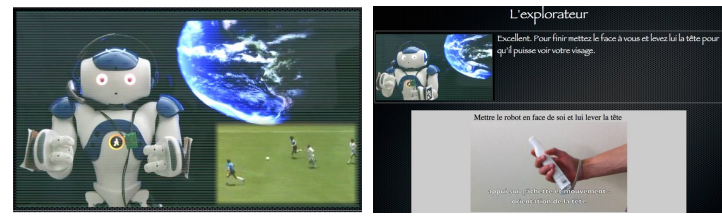

Fig. 15. The story of the game was told through video displayed on our game interface. This display was also used to provide users with step-by-step instructions of the tutorial.

Furthermore, it also allowed us to conduct each test in the same way. Indeed, all participants received the exact same information and instructions through the game interface.

\section{Experimental Protocol}

The experiment took place from June to November 2010 and 107 persons participated in it. Most of them (74) were recruited at Cap Sciences 8 , a science museum in Bordeaux, most of which where visitors. We expected to find, in general, non-expert participants within the public; although, it might have introduced a bias as science museum visitors are probably more receptive to technology. The others (33) were recruited on the campus of Bordeaux University of Technology. We expected to find here participants with a significant technological background and a knowledge of classical interfaces but without any particular robotic knowledge.

Seventy-seven participants were male and 30 were female. The participants were aged between 10 and $76(\mathrm{M}=26.3$, STD =14.8). Among the 107 participants: 32 used the iPhone interface, 27 the Wiimote interface, 33 the Wiimote-laser interface and 15 the gestures interface.

\footnotetext{
${ }^{8}$ http://www.cap-sciences.net/
}

Each participants was asked to follow the following protocol, generated from the result of several pilot studies:

1) Fill in a consent form

2) Fill in a pre-questionnaire

3) Experimentation (robotic game)

- Tutorial

a) Wake up the robot by touching its head

b) Make it move forward

c) Make it turn left and right

d) Turn its head left, right, up and down

e) Make it watch your face (or a ball for the laser interface)

f) Enable the photo mode by touching its head

- Mission

a) Draw the robot's attention toward one randomly chosen object among the 12 other possible objects

b) Take a picture of it

The steps from a) to b) were repeated 4 times

\section{4) Fill in a post questionnaire}

The whole experiment (including the questionnaires) lasted between 20 to 30 minutes per participant.

\section{Measures}

During the experiments, we collected the pictures taken by the robot and analyzed them as described below. Due to the nature of the game interface the images were automatically labeled. Indeed, the participants were asked to show a particular object indicated by the game interface. We also measured the time needed to complete the game and also the intermediate times, i.e. each time a picture was taken.

On top of these measures, we also conducted two questionnaire based surveys inspired by the classical guidelines found in the HRI literature [49][53]. Before the experiment, we administered a demographic survey and a pre-task questionnaire concerning the participant's technological profile (computer, video games and robotic experience) and their attitude toward robotics. After the game, we conducted a post-task survey with the following assertions to which agreement had to be evaluated on a 5 points Likert scale:

- Usability and user's experience

1) It was easy to learn how to use this interface.

2) It was easy to move the robot.

3) It was easy to make the robot look at an object.

4) It was easy to interact with a robot.

5) The robot was slow to react.

6) Overall, it was pleasant to use this interface.

- Robotic game

1) Completing the game was easy.

2) The game was entertaining.

3) I felt like cooperating with the robot.

4) I picture myself playing other robotic games in the future. 


\section{RESUlts}

\section{A. Qualitative analysis of the images}

We first manually sorted the collected pictures, i.e. the visual training examples corresponding to a new object, into three categories: 1) images where the object was entirely seen, 2) images where the object was only partially present, 3) images where the object was not present at all. The objects were defined as "partially visible" as soon as a part was missing. We here considered the object corresponding to the label obtained thanks to the game interface. The figure 16 depicts these results. We performed a one-way ANOVA where the independent variable was the interface used and the dependent variable was the number of object corresponding to the "entirely visible" condition. We found a statistical difference between the four interfaces $\left(F_{3,103}=13.7, p<0.001\right)$. In particular, we can observe that without providing any feedback about what the robot sees to the users (the Wiimote and gestures conditions), the object is entirely visible in only $50 \%$ of the images. The Tukey post-hoc test showed that providing feedback significantly improves this result $(80 \%$ for the laser and $85 \%$ for the iPhone). Furthermore, we can discern that the iPhone interface and in particular its video feedback, prevents users from collecting incorrect learning examples (i.e. where the object is not present) in most cases (only $2 \%$ ).

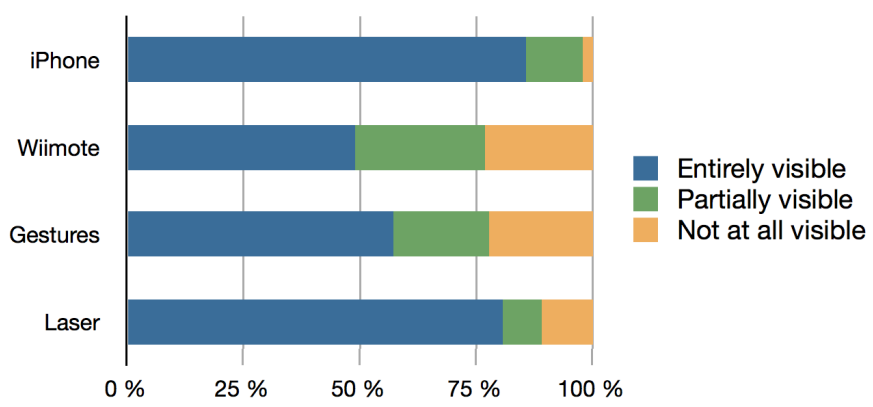

Fig. 16. This figure shows the partition of the collected images into three categories: the object is 1) entirely, 2) partially or 3) not at all visible on the images. We can see that without any feedback (Wiimote or Gesture interfaces) the object was entirely visible in only $50 \%$ of the examples Providing feedback significantly improves this result $(80 \%$ for the laser and more than $85 \%$ for the iPhone).

We also split the images into two subsets:

- big objects: the two posters, the two jerseys and the ball - small objects: the other objects

As we can see in the figure 17, the differences between the interfaces are more accentuated for small objects. In particular, we can see that the lack of feedback led to about a third of examples being incorrect. While the laser feedback improves this result (an error rate of only 20\%), only the iPhone interface seems to really prevent users from providing incorrect learning examples regarding small objects. Finally, we can also observe that users managed to provide rather good examples of the big objects across all the interfaces. Yet, while the objects were almost always entirely visible under conditions where they used the iPhone and Laser interfaces (more than $85 \%$ of the case), they were only partially visible

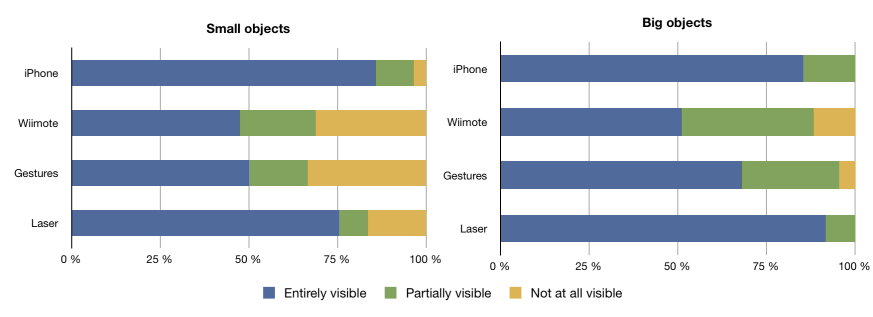

Fig. 17. This figure presents similar charts to the figure 16 but here the collected images were split into two subsets: small and big objects. We can see that the difference between the interfaces is even more accentuated for small objects: with the Wiimote and Gesture interfaces participants failing to provide correct learning examples in about a third of cases.

in about a third of the examples for the Wiimote and Gesture conditions.

\section{B. Quantitative analysis of the images}

We also used the collected images as input training for our learning system in order to have an effective measure of the quality of the learning examples and their impact on the overall performance. As explained above, our learning system is based on a bags of visual words approach. This technique is based on a dictionary used to categorize the features extracted from the images. For our tests we built a dictionary by recording a five minute sequence using the camera of the Nao (about 1000 images) while it was navigating in the living room of our lab. We ensured that none of the furniture or the objects used during the experiments were present during the recording in order to have a dictionary that was not specific to our experimental setup.

We used the following protocol for all the tests:

- We randomly chose $\mathrm{N}$ input images per object collected by users who used a specific interface. Thus, we mixed images taken by several users. As collecting one examples of five objects already took about 20-30 minutes per participant, we could not ask them to collect few examples of each of the twelve objects. This certainly introduced a bias that we tried to counterbalance by randomly selecting images and repeating our tests many times. As shown on the results below, the variability between users (the standard deviation) is rather low. In particular, in most cases the variability among interfaces is larger than the differences among the users of an interface.

- We trained our learning system with these input images.

- We tested our learning on the test database (see below).

- The test was repeated 50 times by choosing a different set of $\mathrm{N}$ training images each time in order to counterbalance the randomization effect.

- The final results are the mean recognition rate and variances of each test.

The test database was built by an expert user who collected 10 examples of each of the objects through the Wizard interface. The images were taken in the same experimental setup as the actual experiment. These examples represented the "best" examples that we could expect as an input. The database was then split in half: the first part was used as a 
"gold" training input while the other half was used as the test data. These "gold" examples were to be used in a similar way as the examples collected with one interface. They were to provide us with an interesting baseline to determine which recognition rate our learning system can achieve with such optimal examples.

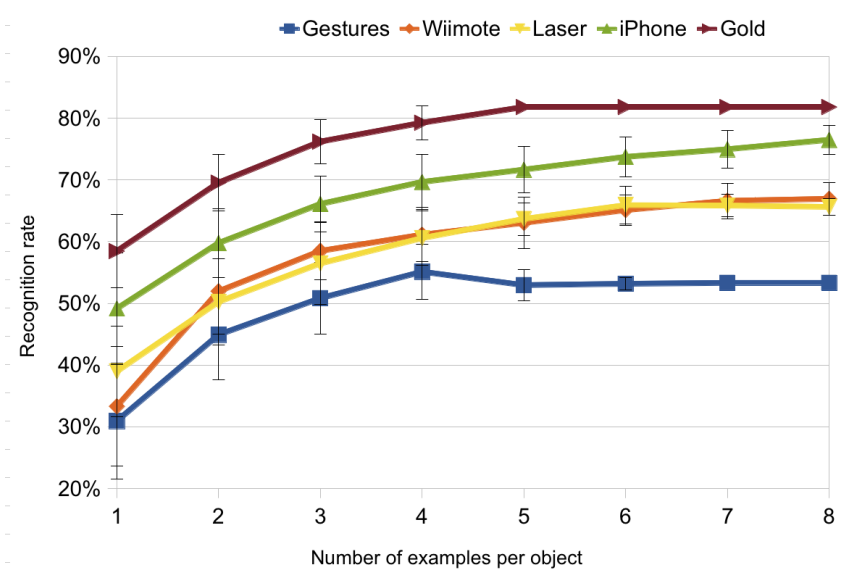

Fig. 18. Recognition rate for all the $\mathbf{1 2}$ objects: This figure shows the impact of the interface on the quality of the learning examples and therefore on the generalization performance of the overall learning system. In particular, we can see that the iPhone interface allows users to collect significantly higher quality learning examples than the other interfaces. Furthermore, it allows even non-expert users to provide the learning system with examples of a quality close to the "gold" examples provided by an expert user.

As shown in figure 18, we notice first that the examples collected with the iPhone interface led to a significantly higher recognition rate than the other three interfaces. In particular, we notice that only three examples collected with the iPhone give as good results as eight examples of any other interface. Furthermore, the iPhone interface seems to allow non-expert users to achieve results close to the results achieved with the gold training after eight examples. We can also see that even with very few good learning examples (such as three or four iPhone examples) we can achieve a rather high recognition rate of 12 different objects (about 70\% correct recognition). Then, we can see that the lowest score was obtained with the Gesture interface. This result can probably be explained by the lack of usability of this interface (see details in the next section).

As in the previous section, we also separated the 12 objects into two categories: big or small. As can be seen in figure 19 . the recognition rate for the big objects is very high (about $90 \%$ ) for all the mediator interfaces. Furthermore, we can see that no significant difference was found between these interfaces. On the other hand, we can see in figure 20 that for the small objects we obtained significantly higher results with the iPhone interface than with the three other interfaces. So, while the interface does not seem to have a very strong impact on the recognition of the big objects, interfaces such as the iPhone interface allows users to obtain a significantly higher recognition rate for small objects, especially with very few learning examples. Those results are coherent with the qualitative results presented earlier.

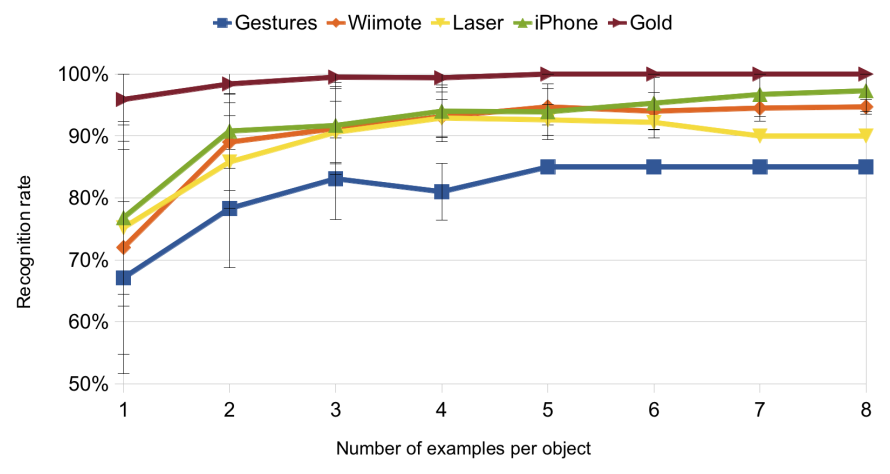

Fig. 19. Recognition rate for the five big objects: We can see that all the mediator interfaces allow users to collect equally good learning examples. So for the big objects the interface does not seem to have a strong impact on the recognition rate.

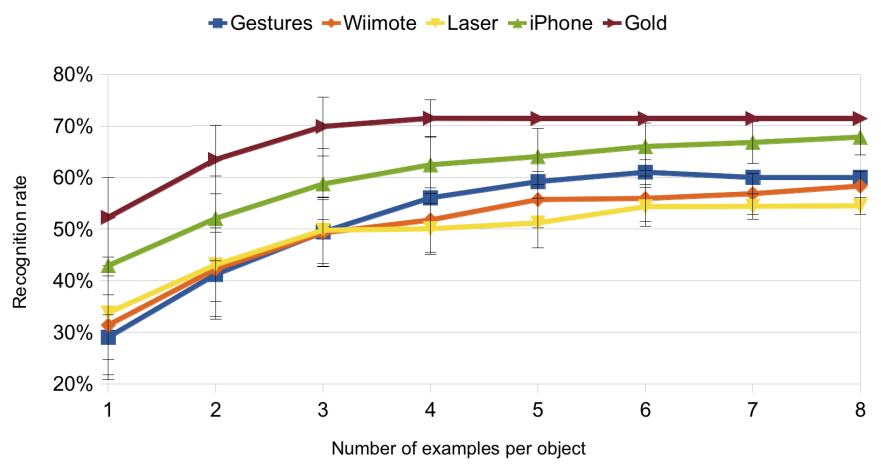

Fig. 20. Recognition rate for the seven small objects: We can see that the iPhone interface allows users to provide higher quality learning examples than the other three interfaces (especially with few learning examples). The other three interfaces gave approximately equal results.

In the above tests, the whole image was used as an input. Thus, the encircling feature of the iPhone interface was not leveraged. We also investigated how encircling impacts the performance of the overall system. As we can see in figure 21, encircling with the iPhone allows us to improve the performance of the system, especially when the system is trained with very few learning examples. In particular, we can see that the recognition rate is between 5 and $10 \%$ higher when trained with less than three encircled learning examples. Yet, we did not find any statistical difference here. Although the experimental environment was cluttered to reproduce a plausible environment, the background was still relatively plain with comparison to many real-world environments where this result would probably be even more important.

We also studied the impact of encircling with the Laser interface. However, we did not find any difference between the two conditions: whole or encircled images. We thus looked in detail at the images collected with this interface and found that in many cases the encircling was correct and should theoretically improve the results. Yet, in many other cases the laser stroke cut the object and so the encircling actually led to a decrease in the quality of the learning examples (as shown on the figure 10 .

These results allow us to show that the interface plays an 


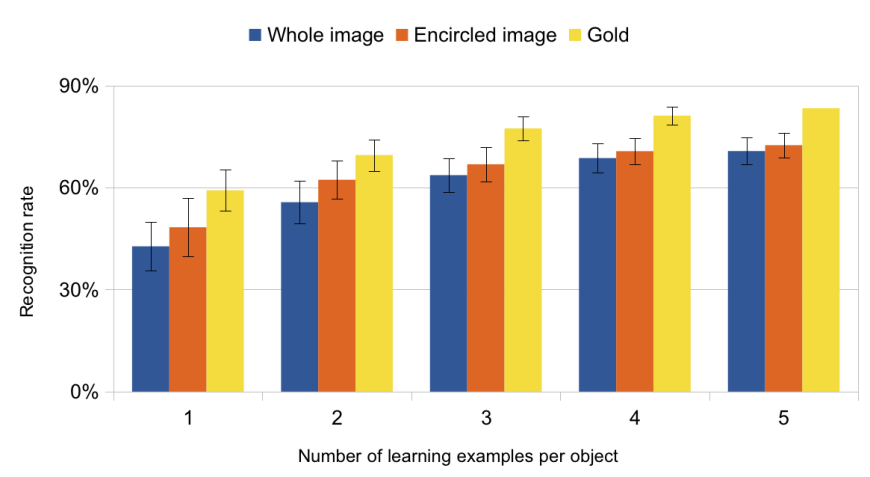

Fig. 21. This figure shows the impact of encircling with the iPhone interface on the recognition rate. As we can see this intuitive gesture allows us to improve the recognition rate, especially when the system is trained with very few learning examples.

important role allowing non-expert users to robustly teach visual objects to a robot. In particular, we showed that the interface has a strong impact on the quality of the learning examples gathered by users. Indeed, we first showed that with simple interfaces such as the Wiimote or the Gesture interfaces that do not provide any feedback to the users, only $50 \%$ of the learning examples collected by users can be considered "good". We also found that encircling improves the recognition rate. Furthermore, we showed that three examples provided by the iPhone interface allow us to obtain a higher recognition rate than eight examples collected with any other interface. These results are particularly important to us as we state that real world users would probably want to give very few learning examples as it could quickly become a tedious task.

We also showed that specifically designed interfaces such as the Laser and the iPhone interfaces, which provides to the users a feedback of what the robot perceives allows nonexpert users to ensure that the object they want to teach is actually visible. While it was expected that providing feedback to the users will help them to collect "apparently" better learning examples, it is very interesting to see that only the examples collected by the iPhone interface led to a very significant improvement of the overall performance of the learning system. Indeed, the Laser and the Wiimote interface gave a rather equal recognition rate. Thus, we can see that the kind of feedback of what the robot perceives also strongly influences the quality of the examples. More precisely, we think that while the Laser interface allows users to know whether an object is visible or not, it does not provide any information on how the object is actually perceived by the robot. For instance, as shown by the examples in figure 22 . many examples were captured either far from the object (so the object was very small in the picture), or the taught object was in the background while other uninteresting objects were in the foreground, etc.

Thus, if one is interested in allowing non-expert users to teach visual objects to a social robot by providing very few learning examples, we think that the interface should really be taken into consideration and specifically designed. In particular, as naive participants seem to have strong incorrect
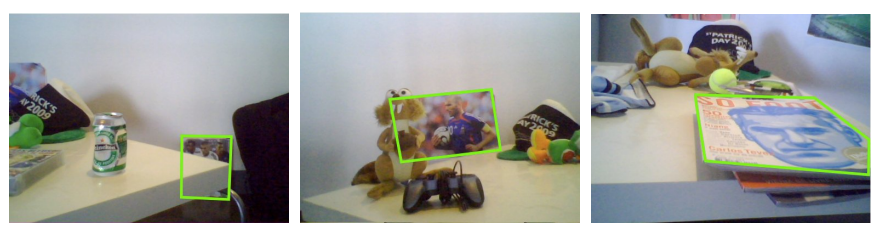

Fig. 22. While the feedback provided by the Laser interface allows users to make sure that the object is visible, it does not help them to realize how the object is actually perceived by the robot. For instance, we can see that the video game on the left is almost entirely occluded by the table. In the center we can see a cluttered foreground in front of the poster of Zidane and finally in the image on the right we can see that the image of the magazine has been taken with an almost horizontal point of view.

assumptions about a humanoid's visual apparatus, we argue that the design of the interface should help users to better understand what the robot perceives but should also drive them to pay attention to the learning examples they are collecting. For instance, the iPhone interface presents, on the screen of the device, the learning example that users encircle and provide to the robot. Thus, the interface naturally forces them to monitor the quality of the examples they collected.

\section{Subjective evaluation of the usability and game experience}

Figure 23 presents the answers to the usability questionnaires. We performed a one-way ANOVA where the independent variable was the interface used and the dependent variable was the answer given in the questionnaires. We found statistical differences for the questions Q1 $\left(F_{3,103}=\right.$ $6.35, p<0.001)$, Q2 $\left(F_{3,103}=2.44, p<0.05\right)$, Q3 $\left(F_{3,103}=6.41, p<0.001\right)$ and Q6 $\left(F_{3,103}=3.38, p<0.05\right)$. The Tukey post-hoc tests showed that the iPhone, Wiimote and Laser interfaces were judged as easier to learn and more practical to move the robot than the Gesture interface. The users also stated that it was easier to make the robot look at an object with the iPhone and Wiimote interfaces. Furthermore, they also judged that overall the iPhone was significantly more pleasant to use than the Laser interface. In particular, during the experiments we observed that the Gesture interfaces led to some misunderstanding while interacting and so participants tended to rush through the experiment.

Figure 24 shows the results for the game part of the questionnaires. The only statistical difference was found for question Q1. We can see that the participants found the game easier when using interfaces based on mediator objects rather than with the gestures interfaces $\left(F_{3,103}=5.17, p<0.005\right)$. The game was judged as entertaining by participants for all conditions. It is also interesting to note that the gestures condition seems to improve the feeling of cooperation with the robot. Similarly, participants seemed to be more willing to play other robotic games with the gestures interface in the future than with the other conditions. However, no statistical difference was found for these results.

It is interesting to note that while the gestures interface was stated as being less usable than the other three interfaces, participants judged that the game was as entertaining with this interface as with the others. To us, this result can be explained by several factors. First, it is important to notice 


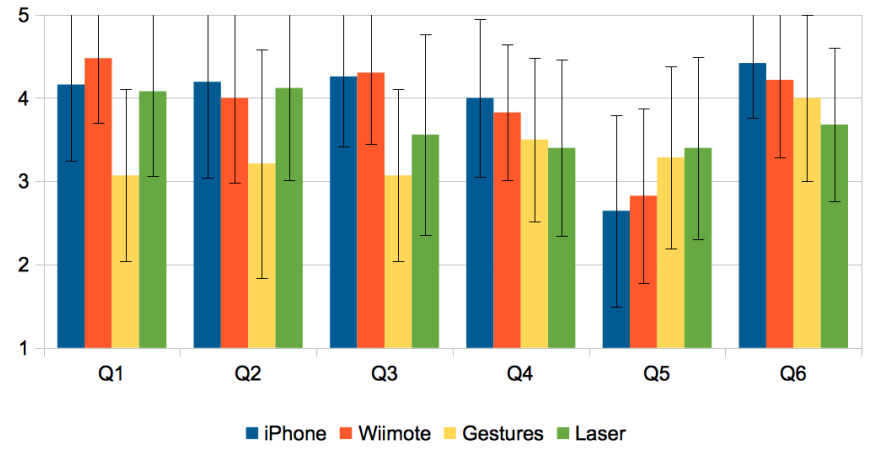

Fig. 23. Usability: Participants found the gestures interface significantly less intuitive and harder to use than the other interfaces. They also stated that the iPhone interface was overall more pleasant than the Laser interface.

Q1: It was easy to learn how to use this interface.

Q2: It was easy to move the robot.

Q3: It was easy to make the robot look at an object.

Q4: It was easy to interact with a robot.

Q5: The robot was slow to react.

Q6: Overall, it was pleasant to use this interface.

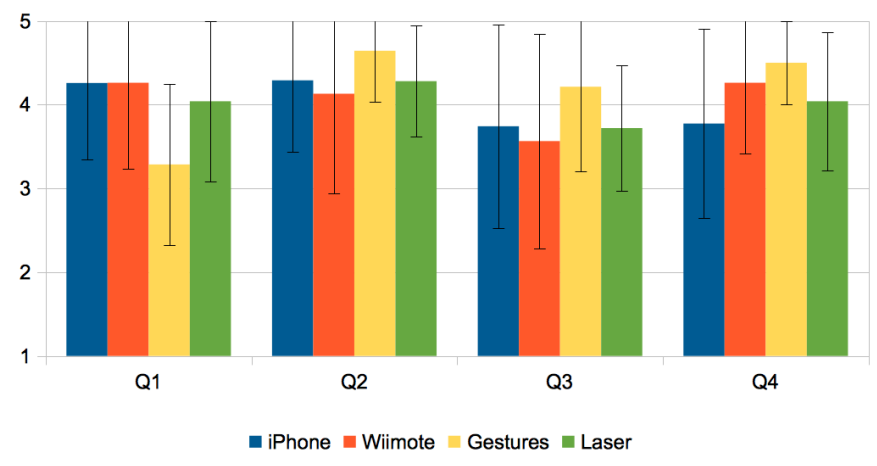

Fig. 24. Robotic game: Our robotic game was stated as entertaining by all participants. They found the game significantly harder with the gestures interfaces but it increased the feeling of cooperation with the robot.

Q1: Completing the game was easy.

Q2: The game was entertaining.

Q3: I felt like cooperating with the robot.

Q4: I picture myself playing other robotic games in the future.

that the participants did not know whether they collected good learning examples or not; it did not influence the user's experience. For instance, users who collected only very bad learning examples could still think that they had successfully finished the game. Second, while interfaces such as the iPhone interface were specifically designed to help users collect good learning examples, it was probably more complicated than necessary for the robotic game. Indeed, users had to monitor the robot, the game interface and the iPhone. Furthermore, it seemed that the interface should be as transparent as possible in order to allow users to entirely focus on the game. Finally, the gestures interfaces seemed to improve the user's feeling that the robot was cooperating with them. We think that this result could be explained by the fact that participants were closer to the robot and that they were trying different gestures to see how the robot reacted and so thereby determining which gestures were better understood. The bias introduce by the Wizard-of-Oz setup also led to a situation where the
Wizard was adapting its behavior to the participants and thus was effectively cooperating with him. Although further studies should be carried out in this direction, our preliminary results about this seem to show that the gestures interface could be interesting if one tries to develop a simple robotic game.

As stated above, we also timed the experience. However, we did not find any significant difference between the different interfaces. Furthermore, for all the results above, no significant differences was found between the participants from the science museum and the participants from the university campus. We also studied other sociological differences (age, gender) without finding any remarkable result.

\section{DISCUSSION AND FUTURE WORK}

We have proposed an integrated system based on a combination of advanced interfaces, visual perception and machine learning methods that allow non-expert users to teach a robotic system how to recognize new visual objects. For experimental convenience, the robotic system was composed of a personal mobile robot and a remote computer which achieves offline signal processing and statistical learning. All the signal processing and statistical learning algorithms used are fast and incremental and it is possible to use them online and onboard a mobile robot [54].

With this system, we have studied the impact of various interfaces based on mediator objects upon the efficiency of robot learning. We have shown that a well designed interface, such as the iPhone, permits the collection of high quality learning examples by non-expert users in realistic conditions and outside of the lab. In particular, providing feedback about what the robot perceives allows non-expert users to collect as good learning examples as expert users who are familiar with the robotic and visual recognition issues.

We have also shown that our interfaces based on mediator objects were judged intuitive and easy to use by participants. Users stated that they were more intuitive and more efficient for teaching new visual objects to a robot than a direct transposition of a human-like interaction based on gestures. This result can be explained by the misconceptions held by non-expert users about what the robot perceives visually. Artefact-based interfaces allow users to understand better what the robot perceives, and thus guide users into providing good learning examples.

In this paper, we also investigated how a robotic game can be used as a framework to conduct real world and large scale user studies. We argue that such an approach allows the design of an experimental setup which engages and motivates users, justifies an a priori artificial task but also has a specific and reproducible protocol. We have also explored the concept and realization of the robotic game which raises interesting questions, especially since robotic games are a relatively unexplored research area [55][56]. Our preliminary result seems to show that users were entertained by our games and were willing to play more robotic games. However, it seems that the game should be rather simple and the interface transparent to allow users to focus on the gameplay. Further studies should be conducted in these directions. 
The experimental setup presented here was designed following some particular design choices. As stated previously, we chose to restrict ourselves to the use of the Nao robot without enhancing its onboard sensors. In our opinion, this robot was representative of some particular characteristics which made it a plausible candidate for the future of personal robotics such as having a humanoid shape, being relatively low-cost and easy to integrate in a domestic environment both from a technological and cultural point of view.

This robot also had some limited sensors which constrained the interaction, but this limitation is currently difficult to avoid in robots targeted for everyday use in the home. As long as robots and humans have different sensorimotor and cognitive apparatuses, robustness issues during interaction will be inevitable, especially if one tries to directly transpose the human-like interaction into the human-robot interaction domain.

Other approaches such as smart environments or ubiquitous robotics have also widely been explored in the literature [57][58]. Using an omnidirectional camera or a set of fixed camera on the ceiling would have changed some of our results and in particular it would have probably facilitated the drawing of attention when using the gestures based interface and thus improving its usability.

Nevertheless, we argue that the interaction problems such as interpreting pointing gestures robustly, knowing what the robot can perceive and making sure that the robot is looking at the object users are showing would still remain despite of these possible enhancements. It is interesting to note that our interfaces could also be combined with external cameras if they are available. For instance, a touch gesture based interface or a laser pointer interface have been combined with ceiling cameras to facilitate drawing a service robot's attention [39][15]. Further experiments should be conducted both in this direction and with other types of robots to evaluate the impact of the robotic setup on our interfaces and on their perception by users.

In the system presented in this paper, the visual objects were automatically labeled, which was made possible by the game context. More precisely, as we used a predefined set of objects during our experiments, they could automatically be associated with a particular symbol provided by the game interface. Such symbolic labels can be easily and surely compared which then allows a direct classification of the different visual examples. This is an important feature as the clustering of the learning examples permits the construction of a better statistical model of the visual object and so a better recognition. However, for more diverse kinds of interaction, we should provide the ability for the users to directly enter a word that they want to associate with the object. Pilot studies have shown that the user would prefer to use vocal words [59]. In future work, we will thus investigate the use of acoustic words associated with visual objects without using an "off-the-shelf" speech recognition system as we argue that they still suffer from robustness problems when used on single words and in uncontrolled conditions. We will in particular investigate the role of the interface for improving the speech recognition, for instance by displaying the $\mathrm{N}$ closest words to the users and allowing them to choose among those possibilities. We will also study how the interface could provide the ability for the humans to incrementally build the complete clusterization of the different learning examples through intuitive and transparent interactions and thus circumvent the issues raised by not using symbolic labels.

Finally, in the experiments described above, we chose to only perform offline visual classification. Indeed, the experiments were already rather complex and time consuming for the users and so we decided not to include the search part in our robotic game to keep the experimental time acceptable for users facilitating the testing of many users. It will be interesting to evaluate our integrated system in a complete scenario including the search of the objects in order to study the entire interaction, and let users have feedback on the learning efficiency of the robot. The robot should itself assess the quality of the learning examples. In such a closed loop scenario, it will be interesting to investigate how the robot could provide feedback to the user regarding the quality of the learning examples collected, or how the robot could use active learning techniques to ask informative questions to the user [60].

\section{ACKNOWLEDGMENT}

The authors would like to thank the Conseil Régional d'Aquitaine and the ERC EXPLORERS 240007 for partially funding this research. The authors would also like to thank Haylee Fogg for her comments and spelling corrections. Finally, they would like to thanks the reviewers for their insightful comments.

\section{VIDEO LINKS}

- Description of the interfaces based on mediator objects : http://youtu.be/vrMsaIj2SDM

- Description of the gestures based interface http://youtu.be/15GOCqXdgQg

\section{REFERENCES}

[1] B. Gates, "A robot in every home," Scientific American, January 2007, http://www.sciam.com/article.cfm?id=a-robot-in-every-home.

[2] T. W. Fong, I. Nourbakhsh, and K. Dautenhahn, "A survey of socially interactive robots," Robotics and Autonomous Systems, 2003.

[3] C. L. Nehaniv and K. Dautenhahn, Eds., Imitation and social learning in robots, humans, and animals: behavioural, social and communicative dimensions. Cambridge University Press, 2004.

[4] A. Billard, S. Calinon, R. Dillmann, and S. Schaal, "Survey: Robot programming by demonstration," Handbook of Robotics, . chapter 59, 2008,2008

[5] A. L. Thomaz and C. Breazeal, "Teachable robots: Understanding human teaching behavior to build more effective robot learners," Artificial Intelligence Journal, vol. 172, pp. 716-737, 2008.

[6] A. Thomaz and C. Breazeal, "Robot learning via socially guided exploration," in Development and Learning, 2007. ICDL 2007. IEEE 6th International Conference on, july 2007, pp. $82-87$.

[7] S. Calinon, F. Guenter, and A. Billard, "On learning, representing, and generalizing a task in a humanoid robot," Systems, Man, and Cybernetics, Part B: Cybernetics, IEEE Transactions on, vol. 37, no. 2, pp. $286-298$, april 2007.

[8] P. Miller, Theories of developmental psychology., 4th ed. New York: Worth, 2001. 
[9] S. Calinon and A. G. Billard, "What is the Teacher's Role in Robot Programming by Demonstration? Toward Benchmarks for Improved Learning," Interaction Studies. Special Issue on Psychological Benchmarks in Human-Robot Interaction, vol. 8, no. 3, 2007. [Online]. Available: http://citeseerx.ist.psu.edu/viewdoc/summary?doi=10.1.1.73. 2276

[10] M. Tomasello, M. Carpenter, J. Call, T. Behne, and H. Moll, "Understanding and sharing intentions: The origins of cultural cognition," Behavioral and Brain Sciences, vol. In Press, 2004. [Online]. Available: http://email.eva.mpg.de/ tomas/pdf/tomasello_et_al2004.pdf

[11] F. Kaplan and V. Hafner, "The challenges of joint attention," pp. 67-74 2004. [Online]. Available: http://cogprints.org/4067/

[12] L. Steels and F. Kaplan, "Aibo's first words: The social learning of language and meaning," Evolution of Communication, vol. 4, no. 1, pp. $3-32,2000$

[13] K. Dautenhahn and J. Saunders, New Frontiers in Human-Robot Interaction, ser. Advances in Interaction Studies. John Benjamins Publishing Company, 2011. [Online]. Available: http://books.google.fr/ books?id=_FlP3ZBhq6oC

[14] P.-Y. Oudeyer, "Developmental Robotics," in Encyclopedia of the Sciences of Learning, ser. Springer Reference Series, N. Seel, Ed. Springer, 2011. [Online]. Available: http://hal.inria.fr/hal-00652123

[15] K. Ishii, S. Zhao, M. Inami, T. Igarashi, and M. Imai, "Designing laser gesture interface for robot control," in Proceedings of the 12th IFIP Conference on Human-Computer Interaction, INTERACT2009, 2009, pp. 479-492.

[16] P. Rouanet, J. Béchu, and P.-Y. Oudeyer, "A comparison of three interfaces using handheld devices to intuitively drive and show objects to a social robot : the impact of underlying metaphors," IEEE International Symposium on Robots and Human Interactive Communications ROMAN, 2009.

[17] P. Rouanet, P.-Y. Oudeyer, and D. Filliat, "An integrated system for teaching new visually grounded words to a robot for non-expert users using a mobile device," in Proceedings of the IEEE-RAS Humanoids 2009 Conference, 2009

[18] P. Rouanet, P. Oudeyer, and D. Filliat, "Using mediator objects to easily and robustly teach visual objects to a robot," in ACM SIGGRAPH 2010 Posters. ACM, 2010.

[19] P. Rouanet, F. Danieau, and P.-Y. Oudeyer, "A robotic game to evaluate interfaces used to show and teach visual objects to a robot in real world condition," in proceedings of the ACM/IEEE Human-Robot Interaction HRI 2011 conference, 2011.

[20] J. Sivic and A. Zisserman, "Video google: A text retrieval approach to object matching in videos," in IEEE International Conference on Computer Vision (ICCV), 2003

[21] D. Filliat, "Interactive learning of visual topological navigation," in Proceedings of the 2008 IEEE International Conference on Intelligent Robots and Systems (IROS 2008), 2008.

[22] K. Mikolajczyk and C. Schmid, "A performance evaluation of local descriptors," IEEE Trans. Pattern Anal. Mach. Intell., vol. 27, no. 10, pp. 1615-1630, 2005.

[23] M. Everingham, L. Van Gool, C. K. I. Williams, J. Winn, and A. Zisserman, "The pascal visual object classes (voc) challenge," International Journal of Computer Vision, vol. 88, no. 2, pp. 303-338, Jun. 2010.

[24] D. K. Roy, "Learning words from sights and sounds: a computational model," Ph.D. dissertation, Architecture and Planning, 1999.

[25] C. Yu and D. H. Ballard, "A multimodal learning interface for grounding spoken language in sensory perceptions," ACM Transactions On Applied Perception, vol. 1, pp. 57-80, 2004.

[26] L. Steels, T. Belpaeme et al., "Coordinating perceptually grounded categories through language: A case study for colour," Behavioral and brain sciences, vol. 28, no. 4, pp. 469-488, 2005.

[27] F. Kaplan, "Talking aibo : First experimentation of verbal interactions with an autonomous four-legged robot," in Learning to Behave: Interacting agents CELETWENTE Workshop on Language Technology, 2000, pp. 57-63.

[28] B. Scassellati, "Mechanisms of shared attention for a humanoid robot," in Embodied Cognition and Action: Papers from the 1996 AAAI Fall Symposium, 1996

[29] D. Perzanowski, A. C. Schultz, W. Adams, E. Marsh, and M. Bugajska, "Building a multimodal human-robot interface," IEEE Intelligent Systems, vol. 16, no. 1, pp. 16-21, 2001.

[30] A. Haasch, S. Hohenner, S. Huwel, M. Kleinehagenbrock, S. Lang, I. Toptsis, G. Fink, J. Fritsch, B. Wrede, and G. Sagerer, "Biron - the bielefeld robot companion," in Proc. Int. Workshop on Advances in Service Robotics Stuttgart Germany 2004 pp. 27-32., 2004. [Online] Available: citeseer.ist.psu.edu/article/haasch04biron.html
[31] D. Roy, "Grounded spoken language acquisition: experiments in word learning," IEEE Transactions on Multimedia, vol. 5, no. 2, pp. 197-209, 2003.

[32] F. Kaplan, Les machines apprivoisées comprendre les robots de loisir. vuibert, 2005.

[33] F. Lömker and G. Sagerer, "A multimodal system for object learning," in Proceedings of the 24th DAGM Symposium on Pattern Recognition. London, UK: Springer-Verlag, 2002, pp. 490-497.

[34] H. Wersing, S. Kirstein, M. Götting, H. Brandl, M. Dunn, I. Mikhailova, C. Goerick, J. J. Steil, H. Ritter, and E. Körner, "A biologically motivated system for unconstrained online learning of visual objects," in ICANN (2), 2006, pp. 508-517.

[35] C. C. Kemp, C. D. Anderson, H. Nguyen, A. J. Trevor, and Z. Xu, "A point-and-click interface for the real world: laser designation of objects for mobile manipulation," in HRI '08: Proceedings of the 3rd ACM/IEEE international conference on Human robot interaction. New York, NY, USA: ACM, 2008, pp. 241-248.

[36] K. Tsui, H. Yanco, D. Kontak, and L. Beliveau, "Development and evaluation of a flexible interface for a wheelchair mounted robotic arm," in HRI '08: Proceedings of the 3rd ACM/IEEE international conference on Human robot interaction. New York, NY, USA: ACM, 2008, pp. $105-112$.

[37] T. W. Fong, C. Thorpe, and B. Glass, "Pdadriver: A handheld system for remote driving," in IEEE International Conference on Advanced Robotics 2003. IEEE, July 2003.

[38] H. Kaymaz, K. Julie, A. Adams, and K. Kawamura, "Pda-based humanrobotic interface," in Proceedings of the IEEE International Conference on Systems, Man \& Cybernetics: The Hague, Netherlands, 10-13 October 2004, 2003

[39] D. Sakamoto, K. Honda, M. Inami, and T. Igarashi, "Sketch and run: a stroke-based interface for home robots," in CHI '09: Proceedings of the 27th international conference on Human factors in computing systems. New York, NY, USA: ACM, 2009, pp. 197-200.

[40] S. Lazebnik, C. Schmid, and J. Ponce, "Spatial pyramid matching," in Object Categorization: Computer and Human Vision Perspectives, A. Leonardis, B. Schiele, S. J. Dickinson, and M. J. Tarr, Eds. Cambridge University Press, Nov. 2009, pp. 401-415. [Online]. Available: http://hal.inria.fr/inria-00548647/en

[41] J. Wang, R. Cipolla, and H. Zha, "Vision-based global localization using a visual vocabulary," in Proceedings of the 2005 IEEE International Conference on Robotics and Automation (ICRA), 2005.

[42] A. Angeli, D. Filliat, S. Doncieux, and J.-A. Meyer, "Real-time visual loop-closure detection," in Proceedings of the International Conference on Robotics and Automation (ICRA), 2008.

[43] H. Bay, A. Ess, T. Tuytelaars, and L. Van Gool, "Speeded-up robust features (surf)," Comput. Vis. Image Underst., vol. 110, no. 3, pp. 346$359,2008$.

[44] D. Nister and H. Stewenius, "Scalable recognition with a vocabulary tree," in Proceedings of the 2006 IEEE Computer Society Conference on Computer Vision and Pattern Recognition - Volume 2, ser. CVPR '06. Washington, DC, USA: IEEE Computer Society, 2006, pp. 2161-2168. [Online]. Available: http://dx.doi.org/10.1109/CVPR.2006.264

[45] J. Ponce, T. L. Berg, M. Everingham, D. Forsyth, M. Hebert, S. Lazebnik, M. Marszałek, C. Schmid, C. Russell, A. Torralba, C. Williams, J. Zhang, and A. Zisserman, "Dataset issues in object recognition," in Towards Category-Level Object Recognition. Springer, 2006, pp. 29-48. [Online]. Available: http://lear.inrialpes.fr/pubs/2006/ PBEFHLMSRTWZZ06

[46] D. Schmalstieg, L. M. Encarnaç ao, and Z. Szalavári, "Using transparent props for interaction with the virtual table," in I3D '99: Proceedings of the 1999 symposium on Interactive $3 D$ graphics. New York, NY, USA: ACM, 1999, pp. 147-153.

[47] M. Hachet, F. Dècle, S. Knödel, and P. Guitton, "Navidget for easy 3d camera positioning from $2 \mathrm{~d}$ inputs," in Proceedings of IEEE $3 D U I$ Symposium on 3D User Interfaces, 2008, best paper award. [Online]. Available: http://iparla.labri.fr/publications/2008/HDKG08

[48] W. Gander, G. H. Golub, and R. Strebel, "Least-squares fitting of circles and ellipses," BIT Numerical Mathematics, vol. 34, no. 4, pp. 558-578, December 1994. [Online]. Available: http: //dx.doi.org/10.1007/BF01934268

[49] M. L. Walters, S. N. Woods, K. L. Koay, and K. Dautenhahn, "Practical and methodological challenges in designing and conducting humanrobot interaction studies," in Proceedings of the AISB'05 Symposium on Robot Companions Hard Problems and Open Challenges in HumanRobot Interaction, University of Hertfordshire. University of Hertfordshire, April 2005, pp. 110-119. 
[50] H. A. Yanco, J. L. Drury, and J. Scholtz, "Beyond usability evaluation: analysis of human-robot interaction at a major robotics competition," Hum.-Comput. Interact., vol. 19, no. 1, pp. 117-149, 2004.

[51] P. Rouanet, P.-Y. Oudeyer, and D. Filliat, "A study of three interfaces allowing non-expert users to teach new visual objects to a robot and their impact on learning efficiency," in Proceedings of the ACM/IEEE Human-Robot Interaction HRI 2010 Conference, 2010.

[52] D. R. Michael and S. L. Chen, Serious Games: Games That Educate, Train, and Inform. Muska \& Lipman/Premier-Trade, 2005.

[53] A. Weiss, R. Bernhaupt, M. Lankes, and M. Tscheligi, "The usus evaluation framework for human-robot interaction," in AISB2009: Proceedings of the Symposium on New Frontiers in Human-Robot Interaction, 2009.

[54] D. Filliat, "A visual bag of words method for interactive qualitative localization and mapping," in Proceedings of the International Conference on Robotics and Automation (ICRA), 2007.

[55] A. G. Brooks, J. Gray, G. Hoffman, A. Lockerd, H. Lee, and C. Breazeal, "Robot's play: interactive games with sociable machines," Comput. Entertain., vol. 2, no. 3, pp. 10-10, 2004.

[56] M. Xin and E. Sharlin, "Playing Games with Robots - A Method for Evaluating Human-Robot Interaction," Education, 2007.

[57] T. Kim, S. Choi, and J. Kim, "Incorporation of a software robot anda mobile robot using a middle layer," Systems, Man, and Cybernetics, Part C: Applications and Reviews, IEEE Transactions on, vol. 37, no. 6, pp. 1342-1348, 2007.

[58] T. Seifried, M. Haller, S. Scott, F. Perteneder, C. Rendl, D. Sakamoto, and M. Inami, "Cristal: a collaborative home media and device controller based on a multi-touch display," in Proceedings of the ACM International Conference on Interactive Tabletops and Surfaces. ACM, 2009, pp. $33-40$.

[59] P. Rouanet and P.-Y. Oudeyer, "Exploring the use of a handheld device in language teaching human-robot interaction," in Proceedings of the AISB 2009 Workshop : New Frontiers in Human-Robot Interaction, 2009.

[60] M. Lopes and P.-Y. Oudeyer, "Active learning and intrinsically motivated exploration in robots: Advances and challenges (guest editorial)," IEEE Transactions on Autonomous Mental Development, vol. 2, no. 2, pp. 65-69, 2010.

Pierre Rouanet Pierre Rouanet obtained his PhD in the FLOWERS team at INRIA and Ensta-ParisTech. He graduated from the University Bordeaux I where he received a degree in computer science. His research topics include the study of the role of human-robot interfaces for teaching new words to a robot, the elaboration of new interfaces for efficient and intuitive joint attention and joint intention between the robot and the human (with touch-pad, wiimote and laser pointer), large-scale user studies with non-specialist users in order to show that interfaces can considerably increase learning efficiency.

Pierre-Yves Oudeyer Dr. Pierre-Yves Oudeyer is responsible of the FLOWERS team at INRIA and Ensta-ParisTech. Before, he has been a permanent researcher in Sony Computer Science Laboratory for 8 years (1999-2007). He studied theoretical computer science at Ecole Normale Supérieure in Lyon, and received his Ph.D. degree in artificial intelligence from the University Paris VI, France. After working on computational models of language evolution, he is now working on developmental and social robotics, focusing on sensorimotor development, language acquisition and life-long learning in robots. Strongly inspired by infant development, the mechanisms he studies include artificial curiosity, intrinsic motivation, the role of morphology in learning motor control, human-robot interfaces, joint attention and joint intentional understanding, and imitation learning. He has published a book, more than 80 papers in international journals and conferences, holds 8 patents, gave several invited keynote lectures in international conferences, and received several prizes for his work in developmental robotics and on the origins of language. In particular, he is laureate of the ERC Starting Grant EXPLORERS. He is editor of the IEEE CIS Newsletter on Autonomous Mental Development, and associate editor of IEEE Transactions on Autonomous Mental Development, Frontiers in Neurorobotics, and of the International Journal of Social Robotics Web: http://www.pyoudeyer.com and http://flowers.inria.fr
Fabien Danieau Fabien Danieau is a PhD student at Technicolor, France and in the VR4I Team at INRIA, Rennes, France. His research interests include haptic interactions, multimedia and user experience. In 2010, he received a MS degree in Cognitics from the Ecole Nationale Supérieure de Cognitique of Bordeaux.
David Filliat David Filliat graduated from the Ecole Polytechnique in 1997 and obtained a PhD in robotics from Université Pierre et Marie Curie in 2001. After 3 years of work on robotic programs for the French armament procurement agency, he is now associate professor at Ecole Nationale Supérieure de Techniques Avancées ParisTech and member of the INRIA and Ensta-ParisTech FLOWERS research team. His main research interest are visual perception, navigation and learning in the frame of the developmental approach to autonomous mobile robotics. 